Matthijs N. Oyaert, Jonas Himpe, Marijn M. Speeckaert, Veronique V. Stove and Joris R. Delanghe*

\title{
Quantitative urine test strip reading for leukocyte esterase and hemoglobin peroxidase
}

https://doi.org/10.1515/cclm-2017-1159

Received December 12, 2017; accepted January 11, 2018; previously published online February 10, 2018

\section{Abstract}

Background: Recently, urine test strip readers have become available for automated test strip analysis. We explored the possibilities of the Sysmex UC-3500 automated urine chemistry analyzer based on complementary metal oxide semiconductor (CMOS) sensor technology with regard to accuracy of leukocyte esterase and hemoglobin peroxidase results. We studied the influence of possible confounders on these measurements.

Methods: Reflectance data of leukocyte esterase and hemoglobin peroxidase were measured using CMOS technology on the Sysmex UC-3500 automated urine chemistry analyzer. Analytical performance (imprecision, LOQ) as well as the correlation with white blood cell (WBC) and red blood cell (RBC) counts (Sysmex UF-5000) were studied. Furthermore, the influence of urinary dilution, haptoglobin, $\mathrm{pH}$ and ascorbic acid as confounders was determined.

Results: Within- and between-run imprecision (reflectance signal) ranged from $1.1 \%$ to $3.6 \%$ and $0.9 \%$ to $4.2 \%$ for peroxidase and $0.4 \%$ to $2.5 \%$ and $0.4 \%$ to $3.3 \%$ for leukocyte esterase. Good agreement was obtained between the UF-5000 for RBCs and peroxidase reflectance $(r=0.843)$ and for WBCs and leukocyte esterase $(r=0.821)$. Specific esterase activity decreased for WBC counts exceeding 100 cells/ $\mu \mathrm{L}$. Haptoglobin influenced the peroxidase activity, whereas leukocyte esterase and peroxidase activities showed a pH optimum between 5.0 and 6.5. A sigmoidal correlation was observed between urinary osmolality and peroxidase activity.

*Corresponding author: Joris R. Delanghe, MD, PhD, Department of Laboratory Medicine, University Hospital Ghent, De Pintelaan 185, 9000 Ghent, Belgium, Phone: 09/332 29 56, Fax: 09/332 49 85,

E-mail: joris.delanghe@ugent.be

Matthijs N. Oyaert, Jonas Himpe and Veronique V. Stove:

Department of Laboratory Medicine, University Hospital Ghent, Ghent, Belgium

Marijn M. Speeckaert: Department of Internal Medicine, University Hospital Ghent, Ghent, Belgium
Conclusions: CMOS technology allows to obtain high quality test strip results for assessing WBC and RBC in urine. Quantitative peroxidase and leukocyte esterase are complementary with flow cytometry and have an added value in urinalysis, which may form a basis for expert system development.

Keywords: hemoglobin peroxidase; leukocyte esterase; urine sediment analysis; urine test strip analysis.

\section{Introduction}

Urinary test strip analysis plays an important role in urinalysis and the value as a screening method has been thoroughly demonstrated [1]. Urine test strip analysis has markedly progressed during the last decades. Although manual reading of urine test strips was common until 2000, more automated analyzers have become available during the last two decades [2]. These instruments offer the possibility to obtain reflectance readings. Test results, therefore, no longer need to be expressed in an ordinal scale. However, many clinical laboratories still adhere to expressing urinary test strip results in an ordinal scale and apparently are not fully aware of the possibilities offered by modern technology. Introduction of modern complementary metal oxide semiconductor (CMOS) technology in strip analysis has allowed very sensitive quantification of test pad colors, as demonstrated for urinary albumin, where quantitative analysis in the micro-albumin range has been reported [3, 4]. Concomitantly, urinary flow cytometry has been introduced in urinalysis, where complex automated analysis of the individual urinary particles has led to major analytical and diagnostic improvements [5-8]. Recently, the UF-5000 automated urine sediment analyzer (Sysmex, Kobe, Japan) has been introduced. This instrument is the third generation of fluorescence flow cytometry analyzer for urinalysis and offers the possibility of accurate counting and differentiation of a broad variety of urinary cells [9].

Leukocyte esterase activity is generally believed to be proportional to granulocyte count, whereas peroxidase activity is strongly correlated with the hemoglobin 
concentration and severity of hematuria $[2,10]$. As has been shown in previous studies, leukocyte esterase and peroxidase reflectance data are useful for verifying flow cytometric data on urinary red blood cell (RBC) and white blood cell (WBC) counts. Comparison between urinary test strip data and flow cytometry can be used for developing expert systems to improve the quality of reported results [2].

WBCs and RBCs and their related parameters leukocyte esterase and peroxidase are key elements in urine sediment analysis. In the present study, we investigated the performance of quantitative urinary test strip analysis and explored the possibilities of an advanced automated urine test strip reader based on the CMOS sensor technology for detection of urinary hemoglobin peroxidase and leukocyte esterase activity. As technical progress in test strip detection has created new possibilities for sensitive analysis, we compared the test strip results with the RBC and WBC flow cytometric counts. In order to analyze the relationship between reflectance data and flow cytometry in detail, the effect of confounding factors (e.g. urinary dilution, haptoglobin, $\mathrm{pH}$ and ascorbic acid) was studied.

\section{Materials and methods}

\section{Sysmex UC-3500 and UF-5000}

Quantitative urine test strip analysis was performed with the automated strip reader UC-3500 (Sysmex, Kobe, Japan). Test strips (Meditape UC-11A, Sysmex, Kobe, Japan; Lot number: AC5004) were used in this study. These strips include reagent pads for ordinal scale reporting of $\mathrm{pH}$, relative density, leukocyte esterase, hemoglobin peroxidase (hemoglobin/myoglobin), nitrite, protein, glucose, ketones, urobilinogen, bilirubin, albumin and creatinine. The intensity of the reaction color of the test pad is detected by measuring the amount of light reflected from the surface of the test pad. A high concentration of analyte corresponds to a low reflectance. The reflectance value, expressed as a percentage within a range from $100 \%$ (white) to $0 \%$ (black), is inversely related to the concentration of the analyte [2]. Refractometry-based specific gravity and clarity are measured by the instrument, and color is rated using a specific algorithm against the blank pad on the strip.

Hemoglobin in urinary specimens is detected by a peroxidase enzyme that is present in the urinary test strip pad and oxidizes tetramethylbenzidine using dimethyldihydroperoxyhexane to obtain a blue dye.

Leukocyte esterase is a useful marker for cells of granulocytic origin [11, 12]. The leukocyte test is based on the classical hydrolysis of an amino acid ester by leukocyte esterase to its corresponding alcohol, which reacts with a diazonium salt to produce a violet azo-dye [13]. Data are expressed in an ordinal scale (as "normal", "negative", "positive" or as nominal concentrations) or quantitative reflectance readings on the outprint (research purpose only). In this study, we used quantitative reflectance readings for statistical analysis [2].

The Sysmex UF-5000 is a third-generation urinary flow cytometer-based analyzer that performs automated microscopic analysis. The UF-5000 is able to recognize count and classify cells by analyzing forward scatter light, side scatter light, side fluorescent light and depolarized side scattered light. Depolarized side scattered light was introduced to improve the sensitivity of crystals and to better discriminate the RBC and crystals [9]. The principle is based on a 488-nm blue laser flow cytometry. The UF-5000 measures urinary conductivity and categorizes the particles based on their size, intracellular structure and staining characteristics. The signals are displayed in scattergrams and histograms, and results are given as counts per microliters as well as counts per high power field. The UF-5000 automatically detects and counts RBC, non-lysed RBCs, white blood cells (WBC), WBC clumps, bacteria, yeast-like cells, crystals, different types of epithelial cells, like transitional or renal tubular cells, sperm cells and casts (hyaline and pathological). Urinary particles that cannot be classified in one of the former categories are counted as "other cells".

\section{Hemoglobin peroxidase and leukocyte esterase evaluation}

Imprecision and limit of quantification: Within-run $(\mathrm{n}=20)$ and between-run $(n=20)$ imprecision was assessed with commercially available control material (UC-control low [LOT: 01601-L] and high [LOT: 01601-H]; Sysmex, Kobe, Japan) as well as on patient samples. Three levels with a low, medium and high leukocyte esterase and hemoglobin peroxidase activity were measured. Intra-run and between-run imprecision were determined in one run during 1 day and on 20 consecutive days with one analysis a day, respectively. Patient samples were only used to determine the within-run imprecision. Reflectance data were used for calculating the $\%$ coefficient of variation $(\mathrm{CV})$ data.

The limit of quantification was determined by measuring a blank saline solution six times and calculated using the following formula: mean reflectance $-10 \times$ standard deviation (SD).

Method comparison: Three hundred and seventy-five freshly collected urinary samples sent to our routine laboratory for urinary sediment analysis were included. All samples were processed within $2-4 \mathrm{~h}$ after arrival in the laboratory. Test strip urinalysis was performed before flow cytometric analysis on the Sysmex UF-5000. Comparison was performed between the hemoglobin peroxidase reflectance data and flow cytometric RBC counts on the one hand and the leukocyte esterase reflectance data and flow cytometric WBC counts on the other hand. The study has been carried out in compliance with the World Medical Association Declaration of Helsinki regarding ethical conduct of research involving human subjects.

\section{Confounding factors}

Urinary dilution: The influence of urinary dilution on peroxidase and leukocyte esterase activity was studied. Eleven samples with final osmolality values ranging from 15 to $800 \mathrm{mOsm} / \mathrm{kg}$ were 
prepared. Urines obtained from healthy volunteers were serially diluted with a $\mathrm{NaCl}$ stock solution with an osmolality of $1600 \mathrm{mOsm} /$ $\mathrm{kg}$ with water. Further, $2 \mathrm{~mL}$ of pooled urine showing hematuria $( \pm 1000 \mathrm{RBC} / \mu \mathrm{L})$ or pyuria $( \pm 1000 \mathrm{WBC} / \mu \mathrm{L})$ was added to $2 \mathrm{~mL}$ of a sample of the osmolality dilution series to obtain a final RBC and WBC count of 500 cells $/ \mu \mathrm{L}$. Peroxidase and leukocyte esterase activity was measured using the UC-3500, osmolality was measured using an OM-6050 Osmo Station ${ }^{\text {TM }}$ analyzer (Arkray, Kyoto, Japan).

Haptoglobin, urinary $\mathrm{pH}$ and urinary ascorbic acid: Haptoglobin interference was studied by spiking a serum pool of patients with an elevated haptoglobin concentration $(2.03 \mathrm{~g} / \mathrm{L})$ to obtain final urinary haptoglobin concentrations ranging from 0.1 to $1.0 \mathrm{~g} / \mathrm{L}$. Haptoglobin concentrations were measured using a BN II Nephelometer [14]. The experiment was performed with serum samples of patients with a Hp 1-1, 2-1 and 2-2 phenotype [15]. Haptoglobin was phenotyped according to Delanghe et al. [16].

The effect of urinary $\mathrm{pH}$ on hemoglobin peroxidase and leukocyte esterase activity was tested by adding a urine specimen with an increased hemoglobin (final reflectance: $11.8 \%$ ) and leukocyte esterase activity (59.9\%) to seven buffered solutions with final $\mathrm{pH}$ values ranging from 4.2 to 9.0 .

Urinary ascorbic acid interference on leukocyte esterase and peroxidase activity was assessed by spiking a urinary ascorbic acid (Merck, Darmstadt, Germany) stock solution (final concentration $1000 \mathrm{mg} / \mathrm{L}$ ) to a blank urinary pool to obtain final urinary ascorbic acid concentrations ranging from 0 to $500 \mathrm{mg} / \mathrm{L}$. The urinary stock solution and pool were both negative for ascorbic acid, peroxidase and leukocyte esterase.

\section{Statistical analysis}

Agreement between automated flow cytometry and test strip data was evaluated by Spearman Rank regression analysis. Multiple regression analysis was used to investigate a model relating leukocyte esterase and hemoglobin field reflectance. All statistical analyses were performed in Medcalc (Software version 15.6.1.; Mariakerke, Belgium).

\section{Results}

\section{Imprecision and limit of quantification}

The within-run (patient and QC samples) and betweenrun imprecision (QC samples) (CV \%) ranged from 1.1\% to $3.6 \%$ and from $0.9 \%$ to $4.2 \%$ for hemoglobin peroxidase and from $0.4 \%$ to $2.5 \%$ and from $0.4 \%$ to $3.3 \%$ for leukocyte esterase, respectively. A summary of the imprecision results is presented in Table 1.

A limit of quantification of 13.6 (mean reflectance: 86.8\%; CV: 0.7\%) and 19.9 (mean reflectance: 97.9\%; CV: $0.4 \%$ ) cells/ $\mu \mathrm{L}$ for hemoglobin peroxidase and leukocyte esterase was determined, respectively.

\section{Method comparison}

\section{Comparison of flow cytometric RBC and hemoglobin reflectance results}

The correlation between RBC data and hemoglobin peroxidase activity is presented in Figure 1. Agreement was acceptable between the flow cytometric RBC data and the UC-3500 hemoglobin reflectance measurements for counts above the upper reference limits (25 RBCs/ $\mu \mathrm{L})$. The following regression equation was obtained:

Table 1: Imprecision results of hemoglobin and leukocyte esterase on the Sysmex UC-3500 analyzer.

\begin{tabular}{|c|c|c|c|c|c|c|}
\hline & \multicolumn{3}{|c|}{ Hemoglobin peroxidase } & \multicolumn{3}{|c|}{ Leukocyte esterase } \\
\hline & $\begin{array}{r}\text { Mean count, } \\
\mathrm{RBC} / \mu \mathrm{L}\end{array}$ & $\begin{array}{r}\text { Mean reflectance, } \\
\%\end{array}$ & $\mathrm{CV}, \%$ & $\begin{array}{r}\text { Mean count, } \\
W B C / \mu L\end{array}$ & $\begin{array}{r}\text { Mean reflectance, } \\
\%\end{array}$ & CV, \% \\
\hline \multicolumn{7}{|c|}{ UC-control material } \\
\hline \multicolumn{7}{|c|}{ Within-run } \\
\hline Low & 13.1 & 93.3 & 1.1 & 23.9 & 96.2 & 0.4 \\
\hline High & 132.5 & 17.0 & 3.6 & 13088 & 60.0 & 2.1 \\
\hline \multicolumn{7}{|c|}{ Between-run } \\
\hline Low & 13.1 & 93.2 & 0.9 & 23.9 & 96.2 & 0.4 \\
\hline High & 137.1 & 16.8 & 4.2 & 10788 & 60.7 & 3.3 \\
\hline \multicolumn{7}{|c|}{ Patient samples } \\
\hline \multicolumn{7}{|c|}{ Within-run } \\
\hline Low & 14.9 & 74.5 & 2.0 & 38.0 & 92.1 & 0.4 \\
\hline Medium & 58.5 & 23.9 & 3.1 & 74.1 & 86.8 & 1.8 \\
\hline High & 3750 & 7.8 & 3.2 & 1381 & 69.3 & 2.5 \\
\hline
\end{tabular}

The results are presented as $\mathrm{CV} \%$. 


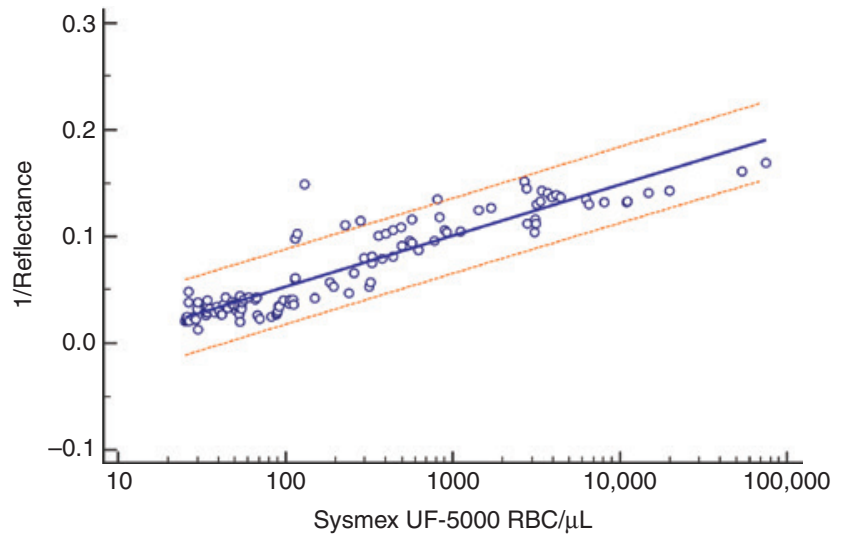

Figure 1: Correlation between RBC counts ( $\mathrm{x}$; flow cytometry) and test strip hemoglobin concentration (y) for RBC counts $>25$ cells $/ \mu \mathrm{L}$ $(n=116)$.

The two outer dashed lines represent the $95 \%$ prediction interval around the regression line (solid line).

$\mathrm{y}(1 /$ reflectance $)=-0.0426+0.0478 \log _{\mathrm{RBC}}($ cells $/ \mu \mathrm{L}) ;$ Spearman $r=0.843 ; p<0.001$ (Figure 1).

\section{Comparison of flow cytometric WBC and leukocyte esterase results}

The correlation between WBC data and leukocyte esterase activity is presented in Figure 2. When urinary WBC counts were higher than the upper reference limit $(25 \mathrm{WBCs} / \mu \mathrm{L})$, the following regression equation was obtained: y $(1 /$ reflectance $)=-0.00724+0.00229 \log _{\mathrm{WBC}}$ (cells $/ \mu \mathrm{L}$ ); Spearman $\mathrm{r}=0.821 ; \mathrm{p}<0.001$ (Figure 2).

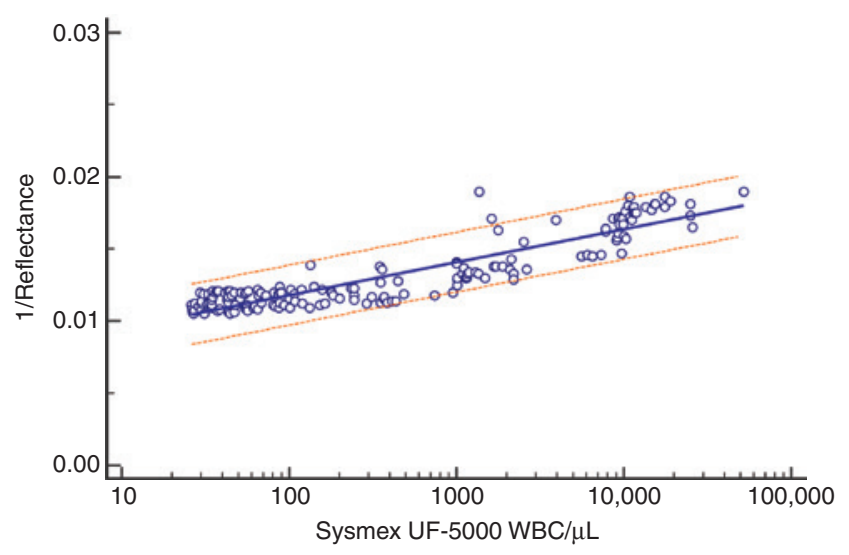

Figure 2: Correlation between WBC counts (x; flow cytometry) and test strip leukocyte esterase concentration (y) for WBC counts $>25$ cells $/ \mu \mathrm{L}(\mathrm{n}=170)$.

The two outer dashed lines represent the $95 \%$ prediction interval around the regression line (solid line).

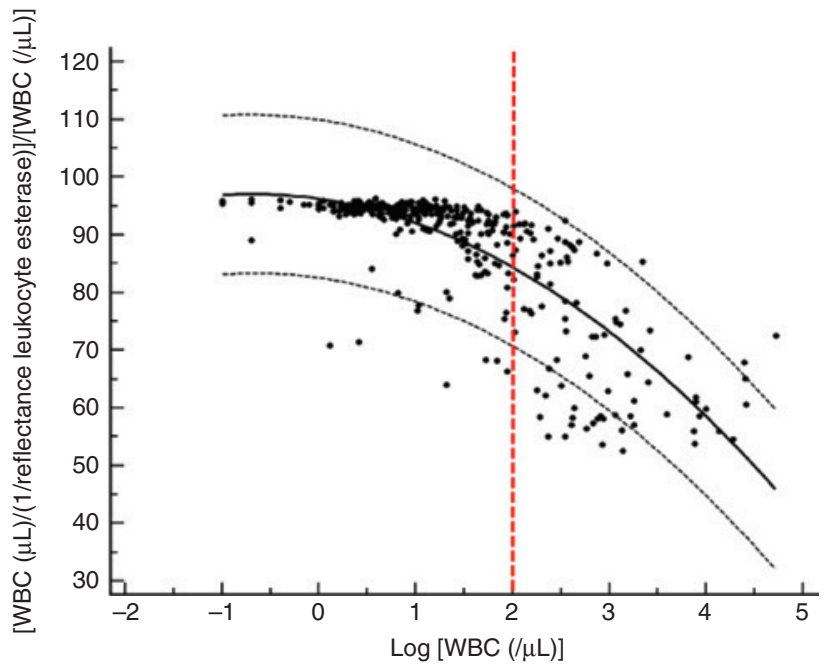

Figure 3: Specific leukocyte esterase activity (activity/WBC) in function of the WBC count $(/ \mu \mathrm{L})$.

The dashed red line indicates the cut-off at which the leukocyte esterase activity decreases. The two outer dashed lines represent the $95 \%$ prediction interval around the regression line (solid line).

When plotting the specific leukocyte esterase activity (esterase activity per counted WBC) versus the WBC count, specific esterase activity dropped $( \pm 12 \%$ per $\log )$ for WBC counts exceeding 100 cells/ $\mu \mathrm{L}$ (Figure 3).

\section{Confounding factors}

\section{Urinary dilution}

Serial dilution of a hypertonic sample $(800 \mathrm{mOsm} / \mathrm{kg})$ resulted in a statistically significant $(\mathrm{p}$-value $<0.05)$ relationship between the osmolality and peroxidase activity (Figure 4A). The polynomial curve is described by the following equation: $\mathrm{y}(1 /$ peroxidase activity $)=0.127+0.027 \times$ $\log (\mathrm{Osm})-0.047 \times \log (\mathrm{Osm})^{2}-0.033 \times \log (\mathrm{Osm})^{3}+0.019$ $\times \log (\mathrm{Osm})^{4}\left(\mathrm{r}^{2}=0.997 ; \mathrm{p}<0.001\right)$. A similar polynomial curve was observed for leukocyte esterase activity y (1/leukocyte esterase activity $)=0.105-0.057 \times \log ($ Osm $)+0.170 \times \log$ $(\mathrm{Osm})^{2}-0.111 \times \log (\mathrm{Osm})^{3}+0.019 \times \log (\mathrm{Osm})^{4} \quad\left(\mathrm{r}^{2}=0.997\right.$; $\mathrm{p}<0.001$ ) (Figure 4B).

\section{Interference of urinary haptoglobin, $\mathrm{pH}$ and ascorbic acid}

Adding haptoglobin (up to final concentration of $1 \mathrm{~g} / \mathrm{L}$ ) resulted in a markedly increase of peroxidase activity. Figure 5 illustrates a linear increase of peroxidase reflectance in function of the molar haptoglobin:hemoglobin 

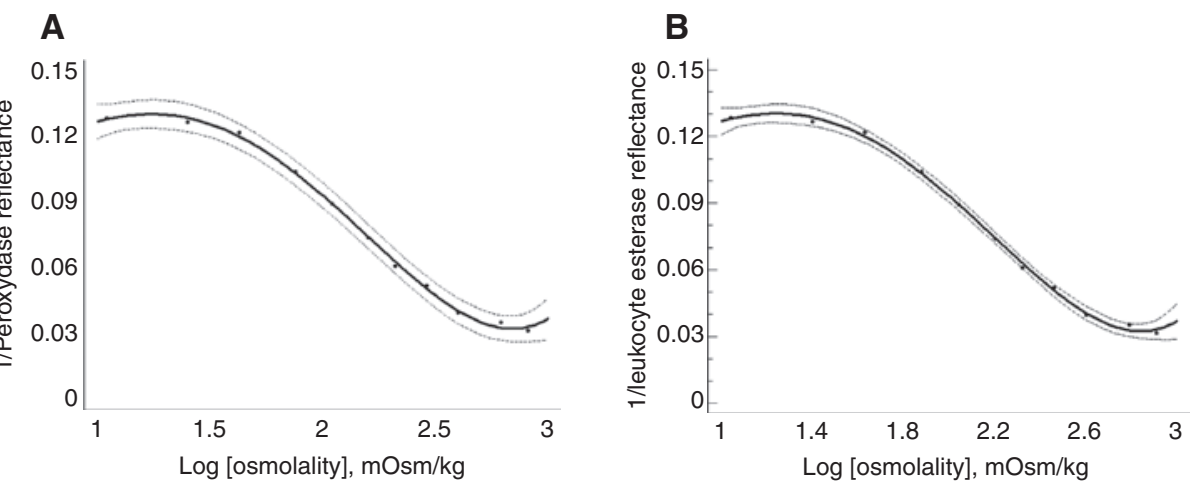

Figure 4: Presentation of the measured osmolality in function of the reciprocal of the peroxidase activity (A) and leukocyte esterase activity (B).

The two outer dashed lines represent the $95 \%$ prediction interval around the regression line (solid line).

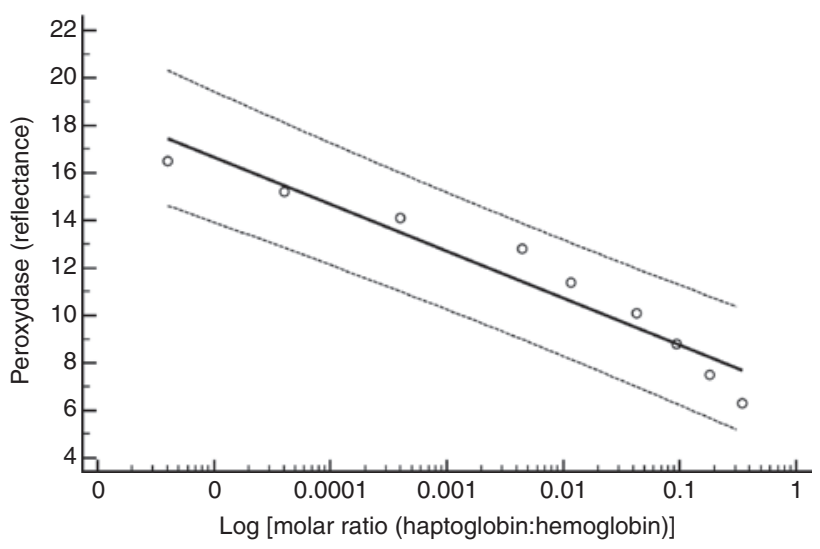

Figure 5: Effect of haptoglobin on peroxidase activity. The haptoglobin concentration is presented as a molar ratio with the hemoglobin concentration. The two outer dashed lines represent the $95 \%$ prediction interval around the regression line (solid line).

ratio: $\mathrm{y}($ reflectance $)=-1.981(\log$ [molar ratio haptoglob in:hemoglobin]) +6.769; Spearman $r=0.932 ; \mathrm{p}<0.001$ ). When repeating the experiment with different haptoglobin phenotypes (Hp 1-1, Hp 2-1 and Hp 2-2), the slopes for the various phenotypes were not significantly different.
Peroxidase and leukocyte esterase activity was found to be maximal in the $\mathrm{pH}$ range between 5.0 and 6.5 and between 6.0 and 7.0, respectively (Figure 6A and B). At higher and lower $\mathrm{pH}$ values, activities of both enzymes decreased.

Addition of ascorbic acid did not result in a significant change neither in peroxidase activity (up to final concentration of $250 \mathrm{mg} / \mathrm{L}$ ), nor in leukocyte esterase activity (up to a final concentration of $200 \mathrm{mg} / \mathrm{L}$ ). At higher ascorbic acid concentrations, statistically significant but clinically irrelevant differences (change in calculated $\mathrm{RBC}<15 \%$ ) between the spiked sample and the blank were observed for peroxidase (from $300 \mathrm{mg} / \mathrm{L}$ ) and leukocyte esterase (from $250 \mathrm{mg} / \mathrm{L}$ ) concentrations (p-value $<0.001$ ).

\section{Discussion}

The landscape of urinalysis has markedly progressed during the last decades. Automatic reading of urine test strips has paved the way for a more quantitative analysis of test strip results $[2,3]$. In the same way, the evolution
A

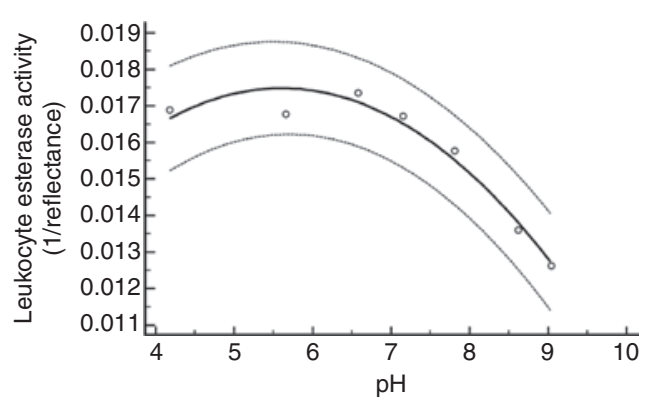

B

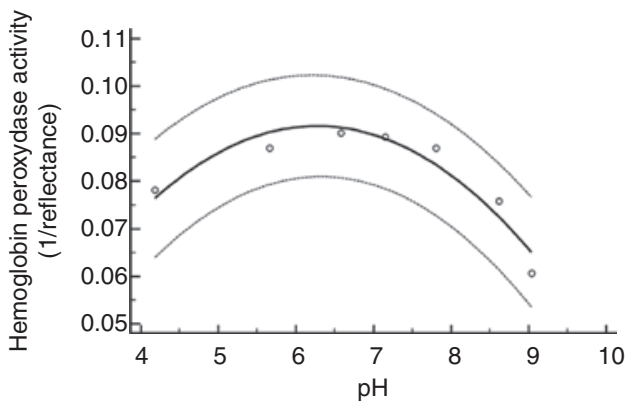

Figure 6: Effect of the $\mathrm{pH}$ on the leukocyte esterase activity (A) and hemoglobin peroxidase activity (B). The two outer dashed lines represent the $95 \%$ prediction interval around the regression line (solid line). 
from microscopy to automated flow cytometry for urine sediment analysis has proven the excellent analytical performance of these analyses [9]. Both evolutions are characterized by a significant decrease in imprecision for both analyses. In the past, poor performance of leukocyte esterase has been reported [17]. In this study, we evaluated the UC-3500 fully automated urine chemistry analyzer and compared the obtained results for hemoglobin peroxidase and leukocyte esterase with $\mathrm{RBC}$ and $\mathrm{WBC}$ counts on the UF-5000, respectively.

Compared to earlier generation automated test strip readers [2], the UC-3500 showed excellent analytical performance results (Table 1). The limit of quantification of the test strip peroxidase and leukocyte esterase corresponds to $13.6 \mathrm{RBC} / \mu \mathrm{L}$ and $19.9 \mathrm{WBC} / \mu \mathrm{L}$, respectively. For both parameters, the limit of quantification roughly corresponds with the upper reference limit of normal [18].

The reciprocal value of the reflectance of the leukocyte esterase signal showed a good correlation with the urinary WBC count $(\mathrm{r}=0.821)$. In comparison with earlier studies [2], the observed correlation coefficient is significantly higher. Because the formulation of the leukocyte esterase test pads is quite similar, the observed improvement cannot be attributed to an improved reagent composition, but to an improved detection of the color signal by the CMOS technology. In severe pyuria (WBC counts $>100$ $\mathrm{WBC} / \mu \mathrm{L})$, specific esterase activity per $\mathrm{WBC}$ appeared to decrease by about $12 \%$ per log. This result can likely be explained by substrate exhaustion in the leukocyte esterase test pad. However, this exhaustion is of minor clinical importance, because it only occurs in urine specimens showing obvious indications of urinary tract infection, where the leukocyte esterase activity clearly confirms the diagnosis.

Furthermore, we evaluated the effect of varying urinary $\mathrm{pH}$ values on leukocyte esterase activity. We demonstrated that leukocyte esterase showed a maximum activity in the $\mathrm{pH}$ range between 5.0 and 6.5. At extremely alkaline $\mathrm{pH}$ values $(>8)$, leukocyte esterase activity decreased. Extremely high urinary $\mathrm{pH}$ ranges can be observed in urinary tract infections with urease producing bacteria, e.g. Proteus spp., in which WBC counts are usually high.

Similarly, an excellent correlation was observed between peroxidase activity and urinary RBC counts. Also for hemoglobin peroxidase, the correlation coefficient was significantly higher as compared to earlier studies [2]. As the composition of the peroxidase test pad is standard based on tetramethylbenzidine, which shows little variation, the improved correlation can also be attributed to an improved electronic reading by the CMOS technology.
As haptoglobin is a protein that is able to bind hemoglobin with a high affinity, and haptoglobin can be present in urine in case of proteinuria [15], the effect of haptoglobin on peroxidase was studied. In agreement with previous observations $[19,20]$, the presence of haptoglobin did affect peroxidase activity of hemoglobin. The various haptoglobin phenotypes showed a similar effect on peroxidase activity. The haptoglobin $\beta$-subunits, which bind to hemoglobin, are identical in the three major haptoglobin phenotypes [15]. These findings may be of importance in case of pronounced proteinuria in combination with hematuria, like is the case in acute glomerulonephritis or diabetic nephropathy [21].

Urinary dilution did affect peroxidase activity. The flow cytometer is able to detect ghost cells (lysed RBCs), which can be observed in the region of low forward scatter on the RBC scattergram [10]. The peroxidase activity showed a sigmoid function in function of urinary osmolality, which is in agreement with observations of RBC lysis in osmotic fragility testing [22]. Similarly, leukocyte esterase activity was affected by urinary dilution, indicating a lysis effect [23]. However, the observed findings are of minor clinical importance as the prevalence of severe hypotonic urine in clinical practice is low.

Various low- and high molecular mass inhibitors have been described in urine $[18,19]$. It is known that reducing substances may lower the peroxidase signal, whereas oxidizing substances may have a positive effect on measured peroxidase activities. Only at extremely high urinary ascorbic acid concentrations ( $>250 \mathrm{mg} / \mathrm{L}$ ), statistically significant, but clinically irrelevant changes in leukocyte esterase and hemoglobin peroxidase activities were observed.

In conclusion, the introduction of the CMOS technology in urine test strip reading has enabled to provide accurate quantitative information about urinary $\mathrm{RBC}$ and $\mathrm{WBC}$ compared to former devices. These quantitative data allow a more precise diagnosis in urinalysis (e.g. added-value in the diagnosis of urinary tract infections and differentiating hematuria). In routine practice, concurrent comparison of flow cytometric data versus test strip results allows to obtain in depth information (e.g. RBC lysis, complexation of hemoglobin to haptoglobin, specific leukocyte esterase activity). The quantitative test strip results may be used to verify flow cytometric results and therefore may form a basis for the development of future urinary expert systems.

Acknowledgments: We are grateful to Sysmex Europe $\mathrm{GmbH}$ for providing the equipment and reagents for conducting the study. 
Author contributions: All the authors have accepted responsibility for the entire content of this submitted manuscript and approved submission.

Research funding: None declared.

Employment or leadership: None declared.

Honorarium: None declared.

Competing interests: The funding organization(s) played no role in the study design; in the collection, analysis, and interpretation of data; in the writing of the report; or in the decision to submit the report for publication.

\section{References}

1. Bonnardeaux A, Somerville P, Kaye M. A study on the reliability of dipstick urinalysis. Clin Nephrol 1994;41:167-72.

2. Penders J, Fiers T, Delanghe J. Quantitative evaluation of urinalysis tests strips. Clin Chem 2002;48:2236-41.

3. Decavele AS, Fiers T, Penders J, Delanghe J. A sensitive quantitative test strip based point-of care albuminuria screening assay. Clin Chem Lab Med 2012;50:673-578.

4. Delanghe J, Himpe J, De Cock N, Delanghe S, De Herde K, Stove V, et al. Sensitive albuminuria analysis using dye-binding based test strips. Clin Chim Acta 2017;471:107-12.

5. Delanghe JR, Kouri TT, Huber AR, Hannemann-Pohl K, Guder WG, Lun $A$, et al. The role of automated urine particle flow cytometry in clinical practice. Clin Chim Acta 2000;301:1-18.

6. Ben-Ezra J, Bork L, McPherson A. Evaluation of the Sysmex UF-100 automated urinalysis analyzer. Clin Chem 1998;44:92-5.

7. Hannemann-Pohl K, Kampf SC. Automation of urine sediment examination: a comparison of the Sysmex UF-100 automated flow cytometer with routine manual diagnosis (microscopy, Test strips, and bacterial culture). Clin Chem Lab Med 1999;37:753-64.

8. Jiménez-Guerra G, Heras-Cañas V, Valera-Arcas MD, RodríguezGrangér J, Navarro JM, Gutiérrez Fernández J. Comparison between urine culture profile and morphology classification using fluorescence parameters of the Sysmex UF-1000i urine flow cytometer. J Appl Microbiol 2017;122:473-80.

9. Previtali G, Ravasio R, Seghezzit M, Buoro S, Alessio MG. Performance evaluation of the new fully automated urine particle analyser UF-50000 compared to the reference method of the Fuchs-Rosenthal chamber. Clin Chim Acta 2017;472:123-30.
10. Langlois MR. Delanghe JR, Steyaert SR, Everaert KC, De Buyzere ML. Automated flow cytometry compared with an automated dipstick reader for urinalysis. Clin Chem 1999;45:118-22.

11. Dumoulin EN, Van Biervliet S, De Vos M, Himpe J, Speeckaert $M M$, Delanghe JR. Faecal leukocyte esterase activity is an alternative biomarker in inflammatory bowel disease. Clin Chem Lab Med 2015;53:2003-8.

12. Kass L. Cytochemistry of esterases. CRC Crit Rev Clin Lab Sci 1979;10:205-23.

13. Skjold AC, Stover LR, Pendergrass JH, Corey PF, Cattell JA, Burkhardt AE, et al. New dip-and-read test for determining leukocytes in urine. Clin Chem 1987;33:1242-5.

14. Fink PC, Römer M, Haeckel R, Fateh-Moghadam A, Delanghe J, Gressner AM, et al. Measurement of proteins with the Behring Nephelometer. A multicentre evaluation. J Clin Chem Clin Biochem 1989;27:261-76.

15. Langlois M, Delanghe J. Biological and clinical significance of haptoglobin polymorphism in humans. Clin Chem 1996;42:1589-600.

16. Delanghe J, Allcock K, Langlois M, Claeys L, De Buyzere M. Fast determination of haptoglobin phenotype and calculation of hemoglobin binding capacity using high pressure gel permeation chromatography. Clin Chim Acta 2000;291:43-51.

17. Mambatta AK, Jayarajan J, Rashme VL, Harini S, Menon S, Kuppusamy J. Reliability of dipstick assay in predicting urinary tract infection. J Fam Med Primary Care 2015;4:265-8.

18. Regeniter A, Haenni V, Risch L, Köchli HP, Colombo JP, Frei R, et al. Urine analysis performed by flow cytometry: reference range determination and comparison to morphological findings, dipstick chemistry and bacterial culture results: a multicenter study. Clin Nephrol 2001;55:384-92.

19. Ottomano C. Tecnologia ed automazione dell' esame urine quantitative. Riv Med Lab 2002;3:60-70.

20. Mattenheimer $\mathrm{H}$, Adams EC. The peroxidase-like activity of the hemoglobin-haptoglobin complex. Z Klin Chem Klin Biochem 1968;6:69-78.

21. Bhensdadia NM, Hunt KJ, Lopes-Virella MF, Tucker JM, Mataria $M R$, Alge JL, et al. Urine haptoglobin levels predict early renal functional decline in patients with type 2 diabetes. Kidney Int 2013;83:1136-43.

22. Palek J, Jarolin P. Hereditary spherocytosis. In: Williams WJ, Beutler E, Erslev AJ, Lichtman MA, editors. Hematology, 4th ed. New York: McGraw-Hill Book Company, 1990:558-69.

23. Marigo S, Bersezio A. Osmotic resistance of leukocytes in patients with onset of diabetes before twenty-five years. Acta Diabetol Lat 1975;12:289-95. 